\title{
Biologics Development and Operations, Molecular and Analytical Development-Bio Separation Department, Bristol Myers Squibb, Hopewell, NJ
}

\author{
Article by Olga Karagiozova \\ PhD, Clinical Research, Texila University, USA \\ E-mail: olgakaragiozova@gmail.com
}

\begin{abstract}
Paracetamol, also known as Acetaminophen is widely used as over the counter or prescription pain reliever and fever reducer that is sold over 50+ different countries. Paracetamol is a nonsteroidal antiinflammatory drug with potent antipyretic and analgesic functions and with very weak anti-inflammatory activity. Paracetamol or Acetaminophen uses diverse brand names, that include not only store brand or generic analgesic but also fever reducers, allergy medicines, medicines for cold, cough, sleeping aids. Other names for Paracetamol include but are not limited to Acetaminodephenol, Acetaminophen, Anacin 3, APAP, Datril, Hydroxyacetanilide, Panadol, Tylenol and etc. ${ }^{(1)}$

At low doses paracetamol is harmless, but it does have direct hepatotoxic potential when taken systematically or as an overdose. It could case acute liver failure as well as injuries to the extrahepatic tissue due to the transient serum aminotransferase elevations. Paracetamol (Acetaminophen) is one of the most commonly taken and used medications in the United States with more than 25 billion doses sold every year. ${ }^{(15)}$

Current article will review the main concepts of paracetamol mechanisms of action, will briefly explain characteristics of pharmacokinetics and pharmacodynamics of the over -the counter analgesic. The metabolic activation of acetaminophen with closer look to starvation, malnutrition, delays in treatment, alcohol, medications and genetics will be outlined as well. Hepatotoxicity along with nephrotoxicity will include pharmacology/pathophysiology, histopathology and associated side effects.
\end{abstract}

Keywords: Paracetamol, acetaminophen, pharmacodynamics, pharmacokinetics, hepatotoxicity.

\section{Concepts}

Paracetamol is an over the counter analgesic and antipyretic medication taken primarily for mild-tomoderate pain and fever as an analgesic and antipyretic agent. It is widely used as over the counter or prescription pain reliever and fever reducer that is sold over 50+ different countries.

Paracetamol can be synthesized from Phenol in three consecutive steps: Nitration of Phenol, Reduction of a nitro group to an Amine and Reduction of a nitro group to an Amine.

Acetaminophen has a central analgesic effect and it is mediated through the activation of descending serotonergic pathways. There are doubts about the primary sites of actions, which could be either the inhibition of the prostaglandin synthesis or an active metabolite function that influences the cannabinoid receptors. The absolute mechanism overall remains undefined, but the primary mechanism is believed to be by the process of inhibition of the cyclooxygenase (COX), with a main effect on the COX-2.

When oral acetaminophen is administered, it is quickly and almost completely absorbed from the gastrointestinal tract, mainly concentrated in the small intestine. The absorption process itself happens by passive transportation. The bioavailability of the paracetamol varies from around $85 \%$ to $98 \%$. Regular release and extended release of the paracetamol are well known, when orally administered.

Paracetamol is a nonsteroidal drug with potent antipyretic and analgesic functions. It is used for the relief of headaches, fever, and other minor pain associated conditions. It is safe in standard doses, does not have 
DOI: 10.21522/TIJAR.2014.04.02.Art006

ISSN: $2520-3088$

anti-inflammatory properties and it is not part of the drug group known as non-steroidal anti-inflammatory drugs (NSAIDs).

One of the leading causes for acute liver failure, accounting for more than 56,000 ER visits, 26,000 needs of medical attention and around 450 deaths per year in USA is the administration and overdose of paracetamol (acetaminophen).

\section{Introduction}

Paracetamol (Acetaminophen) is over-the counter (OTC) analgesic and antipyretic medication (in United States since 1960) taken primarily for mild- to-moderate pain and fever as an analgesic and antipyretic agent.

Paracetamol (Acetaminophen) is usually suggested for the management of different small colds, viral illnesses and bacterial infections, headaches, toothaches, trauma, menstrual cramps, sinusitis and other slight pains that could be easily controlled. In United States during 2011, an intravenous formulation was approved as well for adults and children above age of 2 . The oral dose that is recommended for adults is 660 to 1000 rams every 4 to 6 hours, but it should not exceed 3-4 grams per day. Liquid formulations are also available made for children below certain age, where the concentrations vary from 15 to $100 \mathrm{mg} / \mathrm{mL}$. Multiple generic formulations of acetaminophen are currently available on the market (Tylenol, Anacin Aspirin, Panadol, Neopap) in capsules or tables. In addition to all that, acetaminophen is a common component in many over the counter and prescription mixtures with decongestants, anti-histamines, sleeping aid pills, other analgesics.

\section{Paracetamol}

\section{What is Paracetamol?}

Paracetamol, also known as Acetaminophen is widely used as over the counter or prescription pain reliever and fever reducer that is sold over 50+ different countries. Paracetamol is a nonsteroidal antiinflammatory drug with potent antipyretic and analgesic functions and with very weak anti-inflammatory activity. Paracetamol or Acetaminophen uses diverse brand names, that include not only store brand or generic analgesic but also fever reducers, allergy medicines, medicines for cold, cough, sleeping aids. Other names for Paracetamol include but are not limited to Acetaminodephenol, Acetaminophen, Anacin 3, APAP, Datril, Hydroxyacetanilide, Panadol, Tylenol and etc. ${ }^{(1)}$

\section{Synthesis}

Paracetamol can be synthesized from Phenol in three consecutive steps: ${ }^{(3)}$

\section{Step 1: Nitration of Phenol}

Phenol (hydrobenzene) can react with Sodium Nitrate (NaNO3) - an oxidizing agent to create in the presence of Sulfuric Acid (H2SO4) a mixture of isomers of the Nitrophenol.

\section{Step 2: Reduction of a nitro group to an Amine}

In step two, the oxygen is lost from the Nitro group of 4-Nitrophenol and the hydrogen is added to form the 4-aminophenol.

In this reaction, a catalyst such as palladium or platinum is required.

\section{Step 3: Formation of an Amide}

During this step, 4-aminophenol (amine) reacts with ethanoic anhydride (acetic anhydride) to produce a precipitate of the amide paracetamol/acetaminophen.

\section{Mechanism of action}

Acetaminophen has a central analgesic effect and it is mediated through the activation of descending serotonergic pathways. There are doubts about the primary sites of actions, which could be either the inhibition of the prostaglandin synthesis or an active metabolite function that influences the cannabinoid receptors. The absolute mechanism overall remains undefined, but the primary mechanism is believed to 
be by the process of inhibition of the cyclooxygenase (COX), with a main effect on the COX-2. Thus in the central nervous system, the inhibition of COX enzymes decreases the concentration of prostaglandin E2, which from here lowers the hypothalamic set-points and participates to reduce high fever. The activation of descending inhibitory serotonergic pathways leads to reducing the pain (analgesia). The latest studies demonstrated that acetaminophen inhibits the COX activity in brain homogenates more than those from the spleen. The experiments additionally supported the idea that variant COX enzymes exist and that the paracetamol acts centrally. ${ }^{(4)}$

Recent studies proposed that acetaminophen may be able to work through another mechanism, together with the modulation of the body's endogenous cannabinoid (CB) system. Two autonomous research groups demonstrated an active metabolite of acetaminophen (AM404) shares the ability of CBs to exhibit analgesic activity and to lower body temperature.

Other on-going research studies suggest that the paracetamol produces direct inhibition of the N-methylD-aspartate (NMDA) receptors, blocking substance P-dependent synthesis of Nitric Oxide (NO) through the L-Arginine-nitric oxide pathway and reducing nociception (sensory process that provides the signals that lead to pain). All these proposed pathways are not likely to be neglected; in fact, they may all be components of the series of reactions and responses to acetaminophen management. ${ }^{(4,5,6)}$

\section{Pharmacokinetics}

\section{Characteristics of pharmacokinetics}

\section{Absorption}

When oral acetaminophen is administered, it is quickly and almost completely absorbed from the gastrointestinal tract, mainly concentrated in the small intestine. The absorption process itself happens by passive transportation. The bioavailability of the paracetamol varies from around $85 \%$ to $98 \%$. Regular release and extended release of the paracetamol are well known, when orally administered. ${ }^{(7)}$

\section{Regular release}

24 hours fasting subject who received an acetaminophen $1000 \mathrm{mg}$ as liquid or capsules shows a maximal plasma concentration occurring within 10 to 90 minutes after the ingestion that ranges from $8 \mu \mathrm{g} / \mathrm{mL}$ to 32 $\mu \mathrm{g} / \mathrm{mL}$. An acetaminophen plasma concentration ranges from 1 to $4 \mu \mathrm{g} / \mathrm{mL} 6$ hours after the ingestion approximately.

\section{Extended release}

The In vitro data indicated that the two $650 \mathrm{mg}$ extended release caplets containing a total of $1300 \mathrm{mg}$ of paracetamol, release from $88 \%$ to $95 \%$ or the drug itself within 3 to 5 hours. The average maximal plasma concentration occurred sometimes between 0.5 to 3 hours after administration, and ranged from 6.9 to 14.1 $\mu \mathrm{g} / \mathrm{mL}$.

\section{Distribution}

Paracetamol is widely distributed throughout most of the body excluding the fat. The volume of distribution of paracetamol is around $0.95 \mathrm{~L} / \mathrm{kg}$. Approximately $10 \%$ to $25 \%$ (small part) of the acetaminophen is bound to protein plasma proteins.

\section{Placental barrier}

When urine analysis was performed, it was established that unconjugated paracetamol passes through the placental barrier. When it is given to mothers in the therapeutic doses, the paracetamol crosses the placenta into the fetal circulation as early as the first 30 minutes of digestion. Furthermore, in the fetus, the paracetamol amounts are effectively metabolized. 
DOI: 10.21522/TIJAR.2014.04.02.Art006

ISSN: $2520-3088$

\section{Spinal fluid}

It is found that the peak concentration of paracetamol in the cerebrospinal fluid reaches maximum after 2 to 3 hours of administration. This quick turnaround is due to the low protein binding and low molecular weight that allows for the paracetamol to pass through the blood-brain barrier.

\section{Breast milk}

Current studies established that maternal ingestion of paracetamol in the recommended doses does not present risk to the nursing infants. The measure of paracetamol in the milk range somewhere between $0.1 \%$ to $1.85 \%$ of the actually ingested maternal dose.

\section{Metabolism}

There are three separate pathways for the paracetamol primary metabolism in the liver that are well known and two additional minor pathways that are possibly involved in the paracetamol metabolism:

1. Conjugation with sulfate

2. Conjugation with glucuronide

3. Oxidation via the cytochrome, P450-dependent, mixed-function oxidative enzyme pathway to form a reactive intermediate metabolite.

4. Hydroxylation to form 3-hydroxy-acetaminophen

5. Methoxylation to form 3-methoxy-acetaminophen

\section{Excretion}

The established half-life of acetaminophen in adults is around 2 to 3 hours. It is shorter in range in children and somehow longer in neonates and patients with cirrhosis. The paracetamol is excreted by the body completely by the formation of glucuronide and sulfate conjugates where less than $9 \%$ of the acetaminophen is excreted unchanged in the urine.

\section{Pharmacodynamics}

\section{Characteristics of pharmacodynamics}

Paracetamol is a nonsteroidal drug with potent antipyretic and analgesic functions. It is used for the relief of headaches, fever, and other minor pain associated conditions. It is safe in standard doses, does not have anti-inflammatory properties and it is not part of the drug group known as non-steroidal anti-inflammatory drugs (NSAIDs). When taken in therapeutic doses, paracetamol does not bother the lining of the stomach, does not affect the blood circulation, does not distress the kidneys, nor cause the so called fetal ductus arteriosus. ${ }^{(8)}$

\section{Doses of paracetamol}

Paracetamol is shown to be efficient as a fever reducer at serum concentrations of 10 to $20 \mathrm{mg} / \mathrm{L}$. Orally or rectally the recommended dose is 325 to $650 \mathrm{mg}$ every 4 to 6 hours or $1000 \mathrm{mg}$ every 6 to 8 hours. I.V. dose in adults with weight of 50kg and over is $1000 \mathrm{mg}$ every 6 hours or $650 \mathrm{mg}$ every 4 hours with reaching a maximum dose of $1000 \mathrm{mg}$ and minimum dose of $4000 \mathrm{mg}$ per day. For adults whose weight is under 50 $\mathrm{kg}$, the recommended dose is $15 \mathrm{mg} / \mathrm{kg}$ every 6 hours or $12 \mathrm{mg} / \mathrm{kg}$ every 4 hours with the maximum dose of $15 \mathrm{mg} / \mathrm{kg}$. The minimum dose interval is shown to be 4 hours and a maximum daily dose is established to be $75 \mathrm{mg} / \mathrm{kg}$ per day.

In infants and children that are less than 2 years, the IV form recommendation is 7.5 to $15 \mathrm{mg} / \mathrm{kg} / \mathrm{dose}$ every 6 hours with a maximum daily dose of $60 \mathrm{mg} / \mathrm{kg} / \mathrm{day}$. IV dose for kids from 2 to 12 years recommends $15 \mathrm{mg} / \mathrm{kg}$ every 6 hours or $12.5 \mathrm{mg} / \mathrm{kg}$ every 4 hours. The maximum daily dose is usually around 75 $\mathrm{mg} / \mathrm{kg} /$ day without exceeding the $3750 \mathrm{mg} /$ day dose.

Oral doses recommend from 10 to $15 \mathrm{mg} / \mathrm{kg} /$ dose every 4 to 6 hours without exceeding the 5 doses per every 24 hours. ${ }^{(8)}$ 


\section{Paracetamol and hepatotoxicity}

One of the leading causes for acute liver failure, accounting for more than 56,000 ER visits, 26,000 needs of medical attention and around 450 deaths per year in USA is the administration and overdose of paracetamol (acetaminophen). Even though the acceptable daily dose of paracetamol is around 4 grams a day, chronic digestion of this dose is showing to cause increase of the liver enzymes, even in people who do not undergo with any liver associated diseases. Besides the liver, paracetamol tends to affect the kidneys as well, mainly because of its ability to control the liver's innate detoxification systems. The liver itself uses various enzyme systems that do participate in the metabolism of drugs. $(11,12,13,14)$

\section{Metabolic activation of acetaminophen}

Most of paracetamol is first converted to a toxic metabolite called $\mathrm{N}$-acetyl-p benzoquinoneimine (NAPQI) by phase I CYP (cytochrome P45) enzymes. This middle product then is conjugated with glutathione with the help of phase II enzyme glutathione-S-transferase (GST). The glutathione with time becomes depleted through the process of digestion and NAPQI cannot be sufficiently detoxified. Total hepatic GSH is exhausted by as much as $90 \%$, and this results into the formation of acetaminophen-protein adducts where the metabolite covalently binds to cysteine groups on protein. Increasing levels of NAPQI in the liver starts to cause lipid peroxidation, inactivation of the cellular main proteins, participates in the disruption of DNA metabolism and etc. Furthermore, with the glutathione lost, the oxidative damage increases significantly, and the mitochondria become incapable to produce adenosine triphosphate (ATPcellular energy). This altogether leads to eventual cell death. Similar toxicity can be seen in the kidneys as well and might to some degree lead to acute renal failure.

Overall, a liver necrosis is primarily caused by an overdose.

There are couple of factors that can lower the paracetamol's threshold for overdose and/or increase the possibility of liver failure. They are as follows: Starvation/malnutrition, Delays in treatment, alcohol, other medications, age, and genetics.

\section{Starvation and malnutrition}

Starvation overall is observed to deplete liver glutathione reserves, as well as precursors for other acetaminophen detoxification pathways. This not only increases the toxicity of the acetaminophen dose, but can be also responsible for the toxicity at lower than the average dose. Animal studies demonstrated a protective effect of calories when optimal nutrition is provided to help against experimentally induced acetaminophen toxicity. Increase in sensitivity and acetaminophen toxicity in animals has been associated with low levels/consumption of dietary proteins that are a main source of sulfur-containing amino acids used in the glutathione synthesis.

\section{Delays in treatment}

Delays in treatment of acetaminophen toxic overdose are associated with increased mortality. The common antidote for paracetamol toxicity, $\mathrm{N}$-acetyl cysteine (NAC), begins to lose efficacy in case when administered more than 8-10 hours following acetaminophen overdose.

\section{Alcohol}

Chronic consumption of alcohol lowers the threshold for paracetamol toxicity. This is caused by inducing the CYP (cytochrome P45) enzymes and the depletion of the glutathione reserves. ${ }^{(9)}$

\section{Medications}

Antibiotics, antivirals, anticonvulsants, anti-GERD treatments can increase the toxicity of the paracetamol when administered due to the stimulation of the CUP enzymes, the exhaust of the glutathione stores and the oversaturation of other liver detoxification systems. 
DOI: 10.21522/TIJAR.2014.04.02.Art006

ISSN: $2520-3088$

\section{Genetics}

There are couple of mutations that have been recognized in the phase I and phase II of the detoxification genome/genes necessary for the acetaminophen clearance from the body. There are also genetic mutations observed in the production of the toxic metabolite NAPQI.

\section{Biochemical mechanisms of toxicity}

The sequence of events that lead to hepatocellular death and the formation of acetaminophen protein adduct is poorly understood. One potential mechanism of cell death is that covalent binding to essential cellular proteins results in continuous loss of activity and/or function and potential cell death and cell lysis. Main cellular targets have been proposed to be the mitochondrial proteins, which leads to energy lost, as well as loss of protein involved in the cellular ion control.

\section{Paracetamol and nephrotoxicity}

\section{Pharmacology/Pathophysiology}

Paracetamol is known to be a toxin for both liver and extrahepatic tissues. Extrahepatic manifestations are not well studied as the liver necrosis and are not as often as the hepatotoxicity in terms of occurrence. Overall, renal function disruption occurs in approximately 1 to $2 \%$ of the patients with paracetamol overdose. Some studies show that nephrotoxicity happens more often in children and adolescents when acetaminophen poisoning is present. Usual sign for that is a creatinine elevation of $2.0 \mathrm{mg} / \mathrm{dL}$ in $43-57 \%$ of the 275 patients studied with encephalopathy and coagulatopathy that are secondary results of the paracetamol-induced hepatic failure. It is known that only $1 \%$ of the acetaminophen is excreted unchanged in the urine. With the therapeutic dosage taken by adults, approximately $63 \%$ of the paracetamol is metabolized via glucoronidation and around 34\% through sulfation. When large doses of the drug are ingested, there is more drastic glutathione reduction, excessive metabolite production and increase in toxicity by producing large amounts of reactive species unbound. There are several possible mechanisms of renal toxicity based on both animal and human data. Some of them include the cytochrome P-450 pathway as well as the prostaglandin synthetase and the participation of N-Deacetylase enzymes. Another potential mechanism of paracetamol toxicity is related to the prostaglandin endoperoxidase synthetase (PGES), an enzyme found in the kidneys that activate paracetamol to toxic metabolites, most probably NAPQI. The enzyme N-deacetylase has been also proposed to play role in the paracetamol-induced nephrotoxicity, although this role is still uncertain. ${ }^{(13,21)}$

\section{Histopathology}

Light microscopy of kidney biopsies done to patients with paracetamol toxicity show a tubular epithelial cell necrosis in both, the proximal and distal parts of the tubules. In some cases, there are normal glomeruli and vessels, but with slight damage to the basement membrane. Urinalysis is usually used to distinguish the form of renal insufficiency, such as hepatorenal syndrome (HRS) or pre-renal azotemia. In the case of acute tubular necrosis, there will be granular casts in the urine sediment along with hematuria or pyuria. Sometimes urine sodium, osmolality testing and urinary sediment measurement could also provide additional information for the kidneys current condition. ${ }^{(13,21)}$

Paracetamol associated side effects ${ }^{(17)}$

\section{Rare skin reactions}

Recent findings and FDA waning letters announced that paracetamol has been associated with a risk of rare but very serious skin reactions. Those reactions, usually known as Stevens-Johnson Syndrome (SJS), toxic epitermal necrolysis (TEN) and acute generalized exanthematous pustulosis (AGEP) could often lead to death. Usually those conditions could be associated with skin rash, blisters, and detachment of the upper layers of the skin. These reactions could occur not only to first time use of paracetamol but even when continues treatment is provided. 


\section{Dermatologic}

Dermatologic side effects have been noticed and were recognized as erythematous skin rashes, bullous erythema and purpura fulminans as well.

\section{Gastrointestinal}

A recent study proposed that paracetamol could precipitate and cause acute biliary pain and cholestasis. The mechanisms could be possibly related to the inhibition of the prostaglandins. Gastrointestinal side effects include nausea - around 34\%, vomiting (15\%) as well as acute pancreatitis.

\section{Hematologic}

Hematologic effects are associated with the administration of paracetamol that include but are not limited to rare cases of thrombocytopenia, acute thrombocytopenia, methemoglobinemia with cyanosis associated condition, infusion site pain and peripheral edema.

\section{Hypersensitivity}

Anaphylaxis and fixed drug eruptions were noticed rarely with the administration of paracetamol.

\section{Respiratory}

Respiratory side effects include dyspnea and eosinophilic pneumonia.

\section{Metabolic}

Metabolic side effects were observed with conditions of hypokalemia and metabolic acidosis where the last one was after massive overdose of acetaminophen.

\section{Cardiovascular}

Cardiovascular side effects include hypotension and hypertension were observed even with optimal administration of paracetamol.

\section{Nervous system}

Usual nervous system side effect includes headache, insomnia and fatigue. The headache counts for $10 \%$ of the cases.

\section{Psychiatric}

Psychiatric side effects associated with paracetamol have been reported more often as an anxiety.

\section{Musculoskeletal}

Typical musculoskeletal side effects associated with paracetamol have included muscle spasms and trismus.

\section{Conventional alternatives for paracetamol overdose}

\section{Sulfur containing amino acids}

Amino acids that are rich in sulfur usually are known to support liver health especially after exposure to certain doses of acetaminophen. Daily intake of sulfur containing amino acids could potentially support glutathione levels and are known to protect against drug induced toxicity. ${ }^{(17)}$

\section{Methionine}

Methionine is an essential amino acid that is a precursor to couple of sulfur containing anti-oxidants. It helps with the glutathione level maintenance and is used as a conventional antidote for paracetamol 
DOI: $10.21522 /$ TIJAR.2014.04.02.Art006

ISSN: $2520-3088$

overdose. Some countries do include methionine as part of the acetaminophen formulation in order to protect against accidental intoxication.

\section{N-acetyl cysteine (NAC)}

$\mathrm{N}$-acetyk cysteine is an effectively used in the treatment of acute liver failure due to acetaminophen overdose. It is recommended that every time a paracetamol is taken, at least $600 \mathrm{mg}$ of NAC should be administered to protect against liver toxicity

\section{S-adenosyl methionine (SAMe)}

SAM, as a methionine derivative, is also very important for the synthesis of proteins and phospholipids.

\section{Selenium}

Selenium is a trace element, naturally found in some foods. It acts as a cofactor for numerous enzymes that synthesize the glutathione and therefore participates in the detoxification of paracetamol. Studies show that injecting rats with Selenium around 24 hours before the paracetamol overdose contribute to a significant increase in glutathione levels and lowered levels of ALT and AST.

\section{Curcumin}

It is suggested that curcumin may increase the efficacy of NAC as a paracetamol antidote. It is also proposed that when administered it prevents the microscopic appearance of kidney damage and maintains glutathione levels in comparison to control objects (studies done in rat models).

\section{Co-enzyme Q10 (CoQ10)}

Testing rats before and after paracetamol overdose with CoQ10 showed certain level of liver damage protection. Reduced serum ALT and markers of the oxidative stress were remarkably obvious.

\section{Vitamin C}

High doses of ascorbyl palmitate (not as a free vit $\mathrm{C}$ ascorbic acid) were given and noted to prevent the elevation of serum liver enzymes in mice models. Free vitamin $\mathrm{C}$ did not show protection against hepatic or kidney damage.

\section{Paracetamol awareness}

In 2008, the Acetaminophen Hepatotoxicity Working group in the Center for Drug Evaluation and research (CDER) within the Department of Health and Human Service, FDA (Food and Drug Administration) came up with a report that represents the recommendations ${ }^{(10,18)}$ for FDA interventions that could potentially decrease the number of cases of deliberate and accidental overdose of paracetamol leading to liver and extrahepatic injuries. CDER did recognize that the paracetamol related-hepatotoxicity is a significant public health problem and that certain measurements need to be taken to avoid product overdose and subsequent side effects. Here's list of some of the steps that FDA described and took to steps to implement

a. Enhance public education efforts and make them aware of all the possible effects that an overdose or systematic administration could cause

b. Develop concise clear messages

c. Improve labeling of drugs to reduce intentional overdose in suicide gesture.

d. Improve FDA's own educational efforts

e. Highlight the name of the paracetamol on the display panel of the drug's container

f. Include warnings that when taken above the recommended dose it could cause severe liver injuries and extrahepatic damages.

g. Include warnings for people with liver diseases 
h. Include warning for alcohol use with the medication and incorporate suggested dose when you take more than 3 alcoholic drinks every day while using the product.

i. Put limits on the daily recommended dose for adults for immediate-release formulations to a maximum of $325 \mathrm{mg}$ and a single adult dose of $650 \mathrm{mg}$.

j. Limit table strength for extended-release formulations

k. Limit pediatric liquid formulation

Despite all the efforts, recent research showed that the unintentional and intentional overdoses that are leading cause for severe hepatotoxicity are continuing to take place. In a study published by the Acute Liver Failure Study Group (ALFG) ${ }^{(16)}$, was shown that acute liver failure cases caused by paracetamol overdose increased from $28 \%$ in 1998 to $51 \%$ in 2003 . Severe adverse effects connected to hepatobiliary damage are still reported to FDA with increasing frequency.

\section{Conclusion}

Patacetamol (Acetaminophen) in comparison to other alternative pain reducing medications, such as NSAIDS and narcotics, is relatively safe when used in accordance with the recommended dosage. The ability of paracetamol to cause hepatotoxicity and secondary nephrotoxicity when it is improperly used is not a reason to discourage the public from employing it as a drug.

FDA recent interventions to address the toxic effects and reduce the paracetamol-related hepatotoxicity are only steps for decreasing prospect occurrences of paracetamol (acetaminophen) associated toxicity and avoidance of severe drug related side effects.

\section{References}

[1].13 Sep, 2008; "Paracetamol (Acetaminophen); Mechanisms of action"; Brian J. Anderson PhD, Department of Anaesthesiology, University of Auckland, Auckland, New Zealand; http://onlinelibrary.wiley.com/doi/10.1111/j.1460-9592.2008.02764.x/epdf

[2].2011, Marcia L. Buck, Pharm. D., FCCP, FPPAG; Pediapharm; "Intravenous Acetaminophen Use in Infantts and Children "http://www.medscape.com/viewarticle/742445_2.

[3].Apr, 2000; Br J Clin Pharmacol; Laurie F Prescott " Paracetamol, alcohol and the liver" http://www.ncbi.nlm.nih.gov/pmc/articles/PMC2014937.

[4].December 2003 Laura P. James, Phlip R. Mayeux, Jack A. HInson;” Acetaminophen-induced hepatotoxicity" http://dmd.aspetjournals.org/content/31/12/1499.full.

[5].Dr. Regina M. Botting, William Harvey Research Institute, St. Bartholomew's and Royal London School of Medicine and Dentistry, Charterhouse Square, London EC1 M6BQ, UK "Mechanism of Action of Acetaminophen: Is There a Cyclooxygenase 3?" http://cid.oxfordjournals.org/content/31/Supplement_5/S202.full.

[6].February 2008; Janet Woodcock, M.D. Acting Director, Center for Drug Evaluation and research;" Recommendations for FDA Interventions to Decrease the Occurrence of Acetaminophen Hepatotoxicity “

[7].http://dmd.aspetjournals.org/content/31/12/1499/T1.expansion.html.

[8].http://pubchem.ncbi.nlm.nih.gov/compound/acetaminophen\#section=Boiling-Point.

[9].http://www.ausetute.com.au/paracetamol.html.

[10]. http://www.drugs.com/dosage/acetaminophen.html.

[11]. http://www.drugs.com/sfx/acetaminophen-propoxyphene-side-effects.html.

[12]. http://www.drugs.com/sfx/acetaminophen-side-effects.html.

[13]. http://www.fda.gov/downloads/UCM164898.pdfhttp://www.fda.gov/Drugs/DrugSafety/InformationbyDrugCla ss/ucm165107.htm.

[14]. http://www.lef.org/Protocols/Appendix/Otc-Toxicity/Page-01.

[15]. http://www.rsc.org/learnchemistry/content/filerepository/CMP/00/000/047/Paracetamol_web.pdf.

[16]. http://www.rxlist.com/tylenol-drug/clinical-pharmacology.htm.

[17]. June, 2003; LAURA P. JAMES, PHILIP R. MAYEUX and Jack A. Hinson, , Departments of Pediatrics (L.P.J.) and Pharmacology and Toxicology (L.P.J., P.R.M., J.A.H.), University of Arkansas for Medical Sciences,Little Rock, Arkansas. 
DOI: $10.21522 /$ TIJAR.2014.04.02.Art006

ISSN: $2520-3088$

[18]. March 2008, Volume 4, Issue 1, pp 2-6; “Acetaminophen-Induced Nephrotoxicity: Pathophysiology, Clinical Manifestations, and Management, Maryann Mazer, MD, PharmD, and Jeanmarie Perrone, MD, Department of Emergency Medicine, University of Pennsylvania School of Medicine, Philadelphia, PA; http://downloadv2.springer.com/static/pdf/624/art\%253A10.1007\%252FBF03160941.pdf?token2=exp=1429232436 $\sim$ acl=\%2Fstatic\%2Fpdf\%2F624\%2Fart\%25253A10.1007\%25252FBF03160941.pdf* $\sim$ hmac $=595930926 \mathrm{~b} 556 \mathrm{e} 2635$ dbb2b037eeb0b238e14c27801ec68b2809c4e62756bdd4.

[19]. NIDDK, http://livertox.nih.gov/Acetaminophen.htm. 